\title{
Does Guanfacine Extended Release Impact Functional Impairment in Children with Attention-Deficit/Hyperactivity Disorder? Results from a Randomized Controlled Trial
}

\author{
Mark A. Stein ${ }^{1}$ - Vanja Sikirica ${ }^{2}$ - Margaret D. Weiss ${ }^{3} \cdot$ Brigitte Robertson $^{4}$. \\ Andrew Lyne ${ }^{5} \cdot$ Jeffrey H. Newcorn6
}

Published online: 7 November 2015

(C) The Author(s) 2015. This article is published with open access at Springerlink.com

\begin{abstract}
Background In clinical trials of medications to treat attention-deficit/hyperactivity disorder (ADHD) in children, effects on functional impairment have been less wellstudied than changes in ADHD symptoms.

Objective Data regarding functional impairment were analyzed from a multicenter, double-blind, placebocontrolled study of guanfacine extended release (GXR) in children with ADHD, using the Weiss Functional Impairment Rating Scale-Parent Report (WFIRS-P). The correspondence of changes in WFIRS-P scores with symptomatic and global response to GXR treatment was also examined, with treatment response defined by scores on both the ADHD Rating Scale IV (ADHD-RS-IV) and the Clinical Global Impressions-Improvement Scale (CGI-I).

Methods In this 8-week, double-blind, placebocontrolled, dose optimization study at 47 sites across the USA and Canada, children aged 6-12 years with a diagnosis of ADHD [Diagnostic and Statistical Manual of Mental Disorders, Fourth Edition, Text Revision criteria, and an ADHD-RS-IV score $\geq 28$ and CGI-Severity of
\end{abstract}

Mark A. Stein

mstein42@uw.edu

$1 \quad$ Psychiatry and Behavioral Medicine, Seattle Children's Hospital, 4800 Sand Point Way NE, Seattle, WA 98105, USA

2 GlaxoSmithKline, King of Prussia, PA, USA

3 Department of Psychiatry, University of British Columbia, West Vancouver, BC, Canada

4 Neurovance, Inc., Cambridge, MA, USA

5 Independent statistician, Maidenhead, Berkshire, UK

6 Department of Psychiatry, Icahn School of Medicine at Mount Sinai, New York, NY, USA
Illness Scale score $\geq 4$ at baseline], were randomized 1:1:1 into three groups: GXR AM [GXR $(1-4 \mathrm{mg} /$ day) in the morning, placebo in the evening], GXR PM [placebo in the morning, GXR (1-4 mg/day) in the evening], or twicedaily placebo. Parents rated their children on the WFIRS-P at screening, baseline, the end of dose optimization, and at the final on-treatment assessment.

Results The efficacy population was composed of 333 subjects (GXR AM: $n=107$; GXR PM: $n=114$; placebo: $n=112$ ). At the final on-treatment assessment, there were significant improvements from baseline in the placebo-adjusted difference in least-squares (LS) mean (95\% confidence interval) WFIRS-P Total scores for both GXR treatment groups combined [GXR all-active: $-0.16(-0.25$, -0.07 ), effect size (ES) $=0.448, P<0.001]$ and separately [GXR AM: $-0.15(-0.26,-0.05), \mathrm{ES}=0.417, P=0.004$; GXR PM: $-0.18(-0.28,-0.07), \mathrm{ES}=0.478, P=0.001]$. Significant improvements in WFIRS-P domain scores for Family, Learning and School (including Academic Performance and Behavior in School), Social, and Risky Behavior were found for both GXR cohorts compared with placebo. However, the Life Skills and Self-Concept domain scores of the WFIRS-P did not improve with GXR treatment. Post hoc stratification by responder status revealed that significant $(P<0.001)$ improvements in WFIRS-P Total and all domain scores were associated with symptomatic treatment response in the GXR all-active group.

Conclusions GXR treatment in children with ADHD was associated with reductions in WFIRS-P functional impairment scores compared with placebo, regardless of time of GXR administration. Changes in WFIRS-P scores were congruent with clinical response, as determined by both ADHD symptom reduction and CGI-I scores.

ClinicalTrials.gov identifier NCT00997984. 


\section{Key Points}

The Weiss Functional Impairment Rating ScaleParent Report (WFIRS-P) provides specific information regarding domains of functional impairment in children with attention-deficit/ hyperactivity disorder (ADHD).

Guanfacine extended release (GXR) treatment was associated with improvements in parent ratings of their child's ADHD-related functional impairment, as measured by WFIRS-P, after 8 weeks of treatment.

Improvements on the WFIRS-P were observed for both morning and evening GXR administration.

Parent ratings of functional improvement on WFIRS-P were generally congruent with symptomatic and overall response to GXR treatment, as measured by ADHD Rating Scale IV (ADHD-RS-IV) and Clinical Global Impressions-Improvement Scale (CGI-I) scores, respectively.

The Life Skills and Self-Concept domains of the WFIRS-P did not improve with GXR treatment, which may signal a need for longer trials, or additional or more tailored psychosocial interventions.

\section{Introduction}

Based on the 2011 US National Survey of Children's Health estimates, $11 \%$ of surveyed parents reported that their child (aged 4-17 years, median age 6.2 years) had received a diagnosis of attention-deficit/hyperactivity disorder (ADHD) from a health care provider, with a current prevalence of $8.8 \%$ [1], making ADHD the most commonly diagnosed neurobehavioral disorder of childhood [2]. ADHD is associated with impairments in adaptive functioning and life skills, including problems with socialization, communication, and activities of daily living [3]. Although clinical trials in children with ADHD have historically targeted ADHD symptoms, treatment-related effects may also extend to improvements in functional impairments, such as academic, social, and family functioning, all of which are common reasons for children with ADHD to be referred for treatment [4]. In a study of 314 children referred to an ADHD diagnostic clinic, ADHD symptoms were only moderately correlated with global impairment as measured by a composite Global
Impairment Index score, combining a number of parentand teacher-rated competency scales of behaviors observed at home and school [5]. While it is presumed that symptomatic improvement is generally associated with improved functioning, more work is needed to better understand the relationship between ADHD treatments, changes in symptoms, and specific areas of functional impairment.

The Weiss Functional Impairment Rating Scale-Parent Report (WFIRS-P) is a brief, parent-reported questionnaire developed specifically for children and adolescents with ADHD. The WFIRS-P has been validated using data from seven randomized controlled clinical studies [6]. Confirmatory factor analyses established item-to-scale relationships, with a root mean square error approximation $<0.10$, the cut-off statistic for acceptable model fit; the confirmatory fit index ranged from $0.80-0.88$, which is below the cut-off of 0.9 for acceptable model fit. Cronbach's alpha was $>0.7$ for all six domains, and test-retest reliability was $>0.7$ for all domains other than Risky Activities (intraclass correlation $=0.57$ ). Mean changes in domain scores were significantly different between responder and nonresponder groups in the hypothesized direction. These results were also largely replicated in an independent validation of a translated version of the WFIRS-P in Turkish children [7], and other studies utilizing the WFIRS have noted good reliability of the scale [8,9]. The WFIRS-P has been used to examine functional impairment in observational studies [10], treatment studies of psychoeducational training [11], behavioral therapy in a summer treatment program [11], and clinical trials of lisdexamfetamine dimesylate (LDX) [12] and atomoxetine [13] pharmacotherapy. The WFIRS-P has demonstrated sensitivity in evaluating domains of daily functioning often impaired in ADHD, and in detecting treatment effects of ADHD medications $[12,13]$. In a recent study by Banaschewski et al. [8], treatment with either LDX or osmotic-release oral system-methylphenidate for 7 weeks had a positive impact on functional impairment as measured by the WFIRS-P, with significant placebo-adjusted improvements observed in the Total score as well as in four of the six measured domains (Learning and School, Family, Social Activities, and Risky Activities). Furthermore, in a recent 9-week, head-to-head study of LDX and atomoxetine in children and adolescents with ADHD and an inadequate response to methylphenidate [14], improvements in functional impairment across all six domains were observed with both treatments, with LDX showing superiority over atomoxetine in the Total score as well as in two of the six domains (Learning and School and Social Activities).

There are several non-stimulants that are approved for use in ADHD. Guanfacine extended release (GXR) is a 
selective $\alpha_{2 \mathrm{~A}}$-adrenoceptor agonist indicated for the treatment of ADHD in the USA in children and adolescents aged 6-17 years, both as monotherapy and adjunctive to stimulant medications, and has received a positive opinion from the Committee for Medicinal Products for Human use in the European Union for the treatment of ADHD in children and adolescents. The efficacy and safety of oncedaily morning administration of GXR has been established in children and adolescents with ADHD in several randomized, double-blind, placebo-controlled studies [15-18].

A recent study examined the efficacy and tolerability of once-daily GXR monotherapy administered either in the morning or evening in children aged 6-12 years with ADHD in a randomized, double-blind, placebo-controlled trial [18], and found that administration in either the morning or evening resulted in significantly greater reductions in ADHD symptoms compared with placebo, as measured by the ADHD Rating Scale IV (ADHD-RS-IV), which was the primary efficacy endpoint. The objective of the current substudy (a secondary efficacy endpoint analysis) was to determine whether once-daily GXR (1-4 mg/day) monotherapy, administered in the morning or evening in children aged 6-12 years, impacts overall functional impairment, as measured by the WFIRS-P [18]. An additional goal of this substudy was to examine the effects of treatment on specific domains of functional impairment. Finally, the relationship of functional impairment to clinical improvement was examined in a post hoc analysis of treatment responders versus non-responders by stratifying WFIRS-P scores based upon treatment response (defined by study endpoint scores on the ADHD-RS-IV and the Clinical Global Impressions-Improvement Scale [CGII], a physician-rated measure of global improvement).

\section{Methods}

\subsection{Participants}

Participants were aged 6-12 years with a primary diagnosis of ADHD (combined or hyperactive/impulsive subtype only) as defined by the Diagnostic and Statistical Manual of Mental Disorders, Fourth Edition, Text Revision, based upon the Kiddie-Schedule for Affective Disorders and Schizophrenia-Present and Lifetime version. In addition, subjects were required to have an ADHD-RS-IV Total score $\geq 28$ and a CGI-Severity of Illness score $\geq 4$ at baseline. Key exclusionary criteria included the presence of any current controlled or uncontrolled comorbid psychiatric diagnosis (except oppositional defiant disorder), history of or current suicide risk, and history of or current cardiac abnormalities or primary sleep disorder.

\subsection{Study Design}

In this 8-week, randomized, double-blind, multicenter, placebo-controlled dose optimization study, eligible subjects were randomized 1:1:1 via an interactive web response system to one of the following treatment arms: GXR AM (GXR administered in the morning and placebo in the evening), GXR PM (placebo administered in the morning and GXR administered in the evening), or placebo (placebo administered in the morning and evening) [18]. There was a 5-week dose-optimization period, a 3-week dose-maintenance period, and a 9-day dose-taper period. The starting dose of $1 \mathrm{mg} /$ day was titrated upwards in 1 -mg increments after a minimum of 1 week at the previous dose, based on clinical response and tolerability, up to a maximum of $4 \mathrm{mg} /$ day (Visits 2-7); optimal dose response was defined as the dose which produced $\mathrm{a} \geq 30 \%$ reduction in ADHD-RS-IV Total scores from baseline and a CGI-I score of 1 or 2. After dose optimization, subjects were maintained on their optimal dose for an additional 3 weeks, during which time efficacy and safety were assessed weekly (Visits 8-10). The final on-treatment timepoint was defined as the last non-missing, post-baseline value while on treatment (before dose tapering) with study drug [analogous to Visit 10, last observation carried forward (LOCF)].

\subsection{Assessments}

The primary efficacy measure for the parent study was the change from baseline in ADHD-RS-IV Total symptom score; GXR treatment resulted in lower ratings of ADHD symptoms and significant reductions in ADHD-RS-IV Total scores [18]. This paper focuses on change in functional status with treatment, as measured by the WFIRS-P $[19,20]$.

The WFIRS-P consists of 50 items on which a parent or guardian rates his/her child's functioning; each question is scored on a 4-point Likert scale $[0=$ "never or not at all" to 3 = "very often or very much"; not applicable (NA) is also an option], with higher scores reflecting higher levels of functional impairment. Questions are grouped into six domains: Family, Learning and School (divided into two subdomains: Academic Performance and Behavior in School), Life Skills, Self-Concept, Social Activities, and Risky Activities. Total and domain/subdomain scores, respectively, were the mean item scores across the whole scale or in each domain/subdomain. The WFIRS-P has been reported to have internal consistency and sensitivity to change $[6,7,19,20]$, and shows positive correlations with measures of ADHD symptoms and functioning [8].

In this study, the WFIRS-P was completed at screening (Visit 1), baseline (Visit 2), the end of dose optimization 
(Visit 7), and final on-treatment assessment (Visit 10, LOCF). WFIRS-P domain or Total scores were considered invalid if $>30 \%$ of the item scores used for calculation at baseline were missing or "NA" (with the exception of one question relating to siblings, for which a score of NA could contribute to the number of completed items); the number of NA scores had no effect on the results of the overall analyses since they were so few in number.

Safety evaluations, including assessments of adverse events, vital signs, laboratory measures, and physical examination findings, have been previously reported in detail [18], and therefore are not discussed here.

\subsection{Data Analyses}

The pre-specified exploratory analysis was conducted on all subjects who had taken $\geq 1$ dose of study drug. WFIRS-P analysis included change from baseline in Total, domain, and subdomain scores at Visits 7 and 10, and at final on-treatment assessment. Comparisons included GXR Am versus placebo, GXR PM versus placebo, and both GXR groups combined (GXR all-active) versus placebo. The study was not powered for comparisons between GXR AM and GXR PM. Statistical analysis of the WFIRS-P ratings was performed using an analysis of covariance (ANCOVA) model. No adjustments were made for multiplicity, and least-squares (LS) mean, effect size, and $P$ values were based on type III sum of squares from an ANCOVA model for the change from baseline, including treatment group as a fixed effect and baseline value as a covariate. Effect sizes were measured using Cohen's $d$. Summaries and analyses were performed using the LOCF method.

Functional impairment outcomes were also stratified with response to treatment in a post hoc analysis. Mean WFIRS-P scores were stratified by responder and non-responder status. Response was defined as a $\geq 30 \%$ reduction in ADHD-RS-IV Total score from baseline and a CGII score of 1 or 2 at final on-treatment assessment.

\section{Results}

\subsection{Subject Disposition}

A total of 340 subjects were enrolled; 333 subjects were included in the analysis population. Of these, 221 subjects were administered GXR [all-active group; 107 in the morning (GXR AM), 114 in the evening (GXR PM)] and 112 were administered placebo. Subject demographic and baseline characteristics were similar among treatment groups, and have been described previously in detail [18]. The majority of subjects were male $(70.6 \%)$ and white
$(57.1 \%)$, and the mean age was 9.1 years (range 6-12 years). Among the analysis population, 96.1\% had the combined subtype of ADHD, with a mean time since original diagnosis of 1.7 years. A total of 247 subjects completed the study through Visit 10 [80 (70.8\%) subjects in the GXR AM group, 90 (78.9\%) subjects in the GXR PM group, and $77(68.1 \%)$ subjects in the placebo group].

\subsection{Weiss Functional Impairment Rating Scale- Parent Report}

At baseline, 107, 111, and 109 subjects had complete WFIRS-P data from the GXR AM, GXR PM, and placebo groups, respectively. Three subjects each in the GXR PM and placebo groups from the full analysis set (all subjects who had taken at least one dose of study drug) had missing WFIRS-P data. Mean (standard deviation) baseline WFIRS-P Total scores were similar between the treatment groups: $0.876(0.43)$ for GXR AM; $1.023(0.49)$ for GXR PM; and 0.998 (0.43) for placebo. At the final ontreatment assessment, 97, 100, and 98 subjects had valid WFIRS-P post-baseline data (prior to dose taper) from the GXR AM, GXR PM, and placebo groups, respectively. At the final on-treatment assessment, all GXR cohorts showed significantly greater improvement from baseline in mean WFIRS-P Total scores versus placebo (Table 1). Placeboadjusted differences in LS mean (95\% confidence interval) were GXR AM: $-0.15(-0.26,-0.05)$, effect size $=0.417$, $P=0.004 ;$ GXR PM: $-0.18 \quad(-0.28,-0.07)$, effect size $=0.478, P=0.001$; and GXR all-active: -0.16 $0.25,-0.07)$, effect size $=0.448, P<0.001$.

Baseline WFIRS-P domain and subdomain scores were similar for all treatment groups. However, at the final ontreatment assessment, the mean change from baseline in several WFIRS-P domain/subdomain scores was significantly improved for all GXR cohorts compared with placebo (Fig. 1). These domains/subdomains included Family, Learning and School (both Academic Performance and Behavior in School subdomains), Social Activities, and Risky Activities. The Life Skills domain scores were significantly improved for the GXR all-active group versus placebo at Visit 7, but not at the final on-treatment assessment. Lastly, no significant differences were observed between treatment arms for Self-Concept domain scores.

\subsection{Post Hoc Analyses}

At the final on-treatment assessment, the percentage of responders in the GXR AM (62.5\%), GXR PM (60.4\%), and GXR all-active $(61.4 \%)$ groups was significantly higher than placebo $(30.9 \% ; P<0.001$ for all comparisons). In a further post hoc analysis, a comparison was 





Fig. 1 LS mean change from baseline in WFIRS-P scores at Visit 10 (last observation carried forward) for a GXR AM [GXR (1-4 mg/day) in the morning, placebo in the evening], b GXR PM [placebo in the morning, GXR

(1-4 mg/day) in the evening], and $\mathbf{c}$ GXR all-active; full analysis set. LS mean and $P$ values were based on type III sum of squares from an analysis of covariance model for the change from baseline, including treatment group as a fixed effect and baseline value as a covariate. A negative difference in LS mean (GXR-placebo) indicates a positive effect of the active treatment over placebo. $L S$ least-squares, WFIRS- $P$ Weiss Functional Impairment Rating Scale-Parent Report, $G X R$ guanfacine extended release. $* P<0.05$
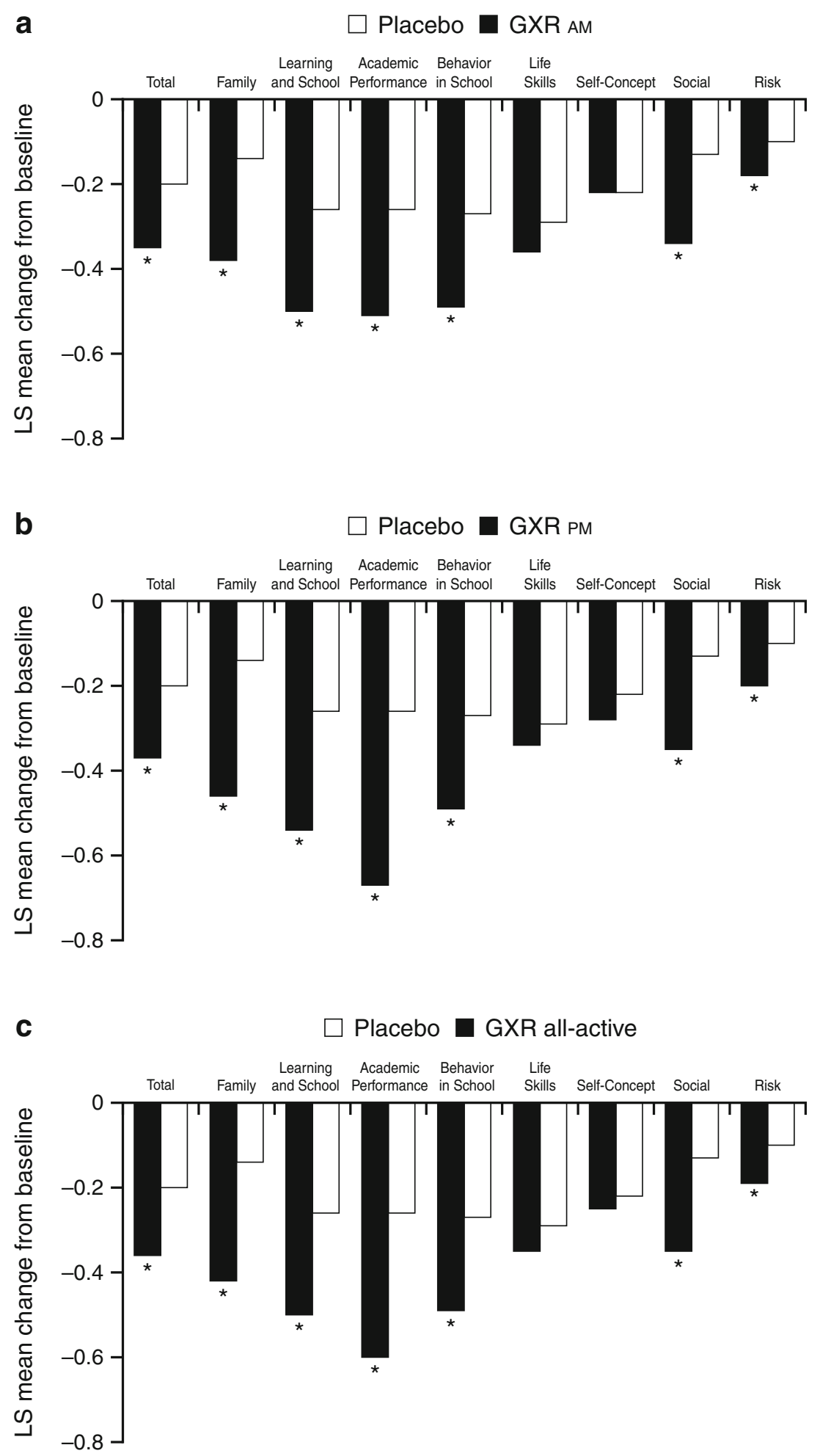

made on the WFIRS-P Total and domain scores for the GXR all-active group responders versus the non-responders. At the final on-treatment assessment, GXR responders demonstrated larger improvements versus non-responders $(P<0.001$ for all scores; Fig. 2).

\section{Discussion}

Children with ADHD display a wide range of functional problems above and beyond academic underachievement [2]. Treatment with GXR, regardless of the time of dosage 


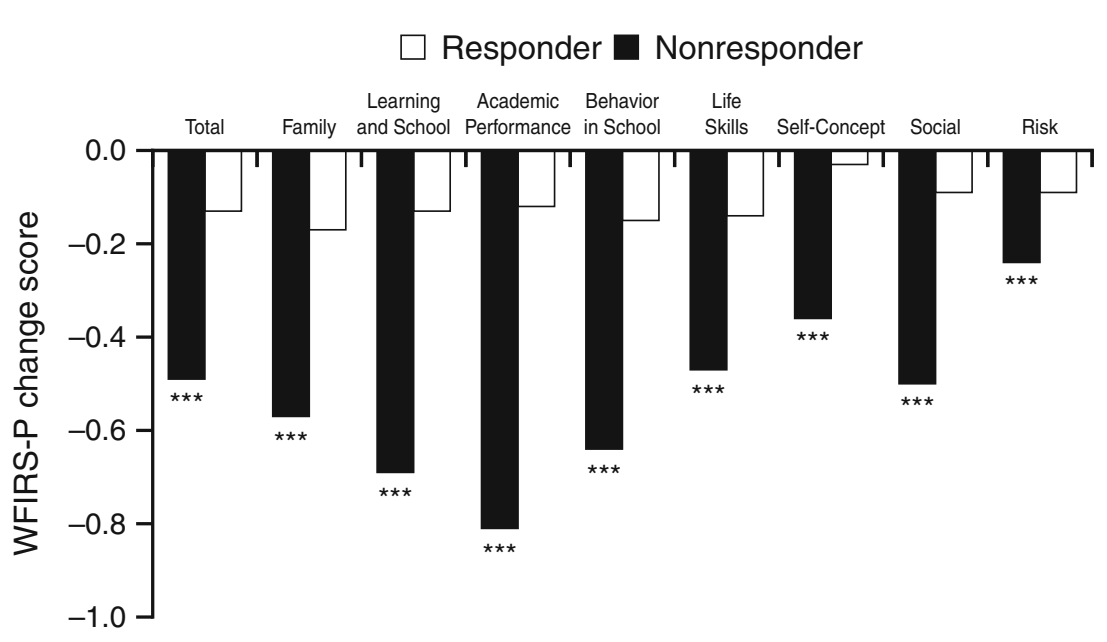

Fig. 2 Mean change from baseline in WFIRS-P scores at Visit 10 (last observation carried forward) by treatment response to GXR treatment; full analysis set (GXR all-active group). Responders are defined as subjects with ADHD-RS-IV Total score reduction from baseline $\geq 30 \%$ and a CGI-I score of 1 or 2. WFIRS-P Weiss

administration (morning or evening), resulted in significant improvements in functional impairment as measured by the WFIRS-P Total score, as well as the majority of domain and subdomain scores. The observed effect size values (Cohen's $d$ ) for GXR AM (0.42) and GXR PM (0.48) WFIRS-P Total scores were in the moderate range [21], and slightly lower than those observed for ADHD symptom improvement with GXR AM (0.75) and GXR PM (0.78) [18]. Notably, improvements in family, school, social, and risk scores were observed with GXR treatment whether administered in the morning or evening ( $P<0.05$ for both). Within the Learning and School domain, subdomain scores for both Academic Performance and Behavior in School also significantly improved compared with placebo (effect sizes ranged from $0.39-0.41$ for the all-active group). The effect sizes for these subdomain scores were expectedly smaller than the treatment effect size seen when using the WFIRS-P Total score, as the subdomain scores were based on fewer WFIRS-P items. These results suggest that, on the whole, treatment with GXR resulted in significant reductions in functional impairment after 8 weeks of treatment when compared with placebo.

In contrast to the specific domains of impairment measured by the WFIRS-P that improved with treatment, both the Life Skills and Self-Concept domain scores did not differ from placebo at the final on-treatment assessment. While the Life Skills domain scores significantly improved for the GXR all-active group versus placebo at Visit 7, it remains unclear why this improvement was not observed at the final on-treatment assessment. These domains may be less sensitive to measuring change, or require more intensive or longer treatment for change to occur. It may also be that Self-Concept in clinical trial subjects improves for all
Functional Impairment Rating Scale-Parent Report, GXR guanfacine extended release, ADHD-RS-IV Attention-Deficit/Hyperactivity Disorder, CGI-I Clinical Global Impressions-Improvement Scale. $* * * P<0.001$

regardless of treatment, given that these subjects often receive support and encouragement from trial staff for study participation and completion.

More research is needed to understand if improvement in the above domains is particularly resistant to change or measurement in a short-term trial. In a small single-center, open-label, non-randomized study of children with ADHD treated with atomoxetine $(n=21)$ [13], significant improvement on the WFIRS-P Self-Concept domain was not observed after 2 months of treatment. However, improvement in Self-Concept was observed after 6 months of treatment, and significant improvement in the Life Skills domain was detected at both 2 and 6 months after treatment; no significant improvements were observed in the Social Activities and Risky Activities domains. Thus, results from both the previous and current studies suggest that the time course for treatment-related functional changes may vary across domains of impairment, and also in relation to onset of symptom change. Perhaps the lack of GXR treatment-related improvement on particular WFIRS$\mathrm{P}$ domains may signal the need for additional or more tailored psychosocial interventions targeting specific life skills and self-concept. It remains to be determined whether or not the GXR-related improvements observed for all other WFIRS-P domains would become larger with a treatment period $>8$ weeks or if they would appear within the Life Skills or Self-Concept domains; this requires further study.

Reductions in functional impairment were related to symptomatic improvement and global response. Of note, among responders, the degree of functional change reported on the WFIRS-P was found to be comparable between those on GXR and placebo. This suggests that perceived improvement in ADHD status is congruent with 
improvements in functional impairment regardless of the intervention, and even whether active medication is received. Among those on GXR, there was separation between responders and non-responders, and the effect sizes for improvement in WFIRS-P Total and domain scores were in the moderate to large range. Nevertheless, the effect size for functional improvement, even where demonstrated, was lower than that for ADHD symptoms. This difference is not surprising given that pharmacologic treatments are specifically targeted to improve symptoms; improvements observed in functional outcomes are a welcome secondary development, but are not necessarily expected. Moreover, given that function is a more distal concept, and that the inclusion/exclusion criteria of the current trial were created to identify subjects with ADHD symptoms and not functional impairment, it is expected that there will be a lesser impact on function in this population. What is remarkable is the strength of functional impairment that does occur in multiple studies and in most (but not all) domains. The fact that some domains do not improve with treatment suggests that when improvement does occur, it is not simply a function of the correlation between symptom measures and functional measures. It is possible that functional impairment in some areas improves more with other forms of treatment (e.g., psychosocial [11, 22]), whereas symptoms of ADHD may decrease more substantially with psychopharmacologic interventions. Regardless, both symptom and impairment measures are important outcomes for treatment and, although correlated, are not the same.

The results of the current study are similar to those obtained by Hervas et al. [23] in a recent phase III study of dose-optimized GXR treatment in children and adolescents with ADHD, which found that subjects on GXR showed significant placebo-adjusted improvements in both the WFIRS-P Learning and School domain and Family domain (study key secondary endpoints) at the last on-treatment study visit prior to dose tapering (effect sizes $=0.42$ and 0.38 for each domain, respectively). Also, comparatively larger magnitude placebo-adjusted improvements on the WFIRS-P have been shown in subjects with ADHD after treatment with the stimulant LDX (effect size for WFIRS-P Total score $=0.924$ ) [8] These prior findings, in combination with those of the current study, suggest that treatment with both non-stimulants and stimulants has a significant impact on functional impairment outcomes, as measured by the WFIRS-P.

The clinical relevance of identifying and targeting functional impairment is substantial. At home, ADHD may negatively impact a child's ability to follow family routines, straining relationships with parents and siblings [24, 25]. At school, children with ADHD are more likely to display academic difficulties than those without ADHD [26], and these difficulties are associated with decreased academic achievement and decreased vocational achievement later in life [27, 28]. Recent evidence using a large registry database also highlighted an increased incidence of accidental injury among children with ADHD; in children treated with ADHD drugs compared with matched untreated children, the prevalence of injuries was reduced by $31.5 \%$ at age 10 years and $43.5 \%$ at age 12 years [29]. Results from this study further suggest that treatmentrelated improvements may extend beyond core symptoms to include improvements in functional impairment, which presumably may lead to improved academic performance, better parent-child relationships, and decreased incidence of accidental injury. It remains to be seen, however, if the improvements seen in short-term trials, such as the current one, can persist and be maintained in the longer term.

A number of study limitations should be noted. First, both the WFIRS-P and ADHD symptoms measures are largely based on parent report. Because they are observational only, the ratings may be subject to parental bias compared with more objective measures (e.g., grades, number of play dates). In addition, because both scales are completed by the same rater, there is potential for confounding; it would be important to determine if parent ratings of functional improvement are in agreement with scales completed by other informants, such as teachers. However, parent reports often drive referral for treatment, and identifying parentreported improvement in functional status via the WFIRS-P represents some reassurance that improvement in symptoms translates to functional status; in this sense, using a measure of functional status together with traditional measures of symptomatic and overall response represents a step forward. Another limitation is that this study was not adequately powered to directly compare the GXR AM and GXR PM groups, and the statistical tests conducted were not corrected for multiple comparisons, allowing for the possibility of type I error. Third, the subjects predominately had combined type ADHD with moderate to severe symptom burden, which may limit the generalizability of the study results to children with less severe ADHD or those with the predominantly inattentive presentation. Finally, while these results suggest a significant relationship between improvements in symptoms and improvements in functional impairment, future research should more precisely examine the relationship between symptomatic and functional domains, i.e., whether symptom change mediates improvement in functional status, and whether there are baseline factors that moderate these relationships.

\section{Conclusions}

Once-daily GXR monotherapy administered in the morning or evening was associated with reductions in functional impairment in children with ADHD, as assessed by the 
WFIRS-P scale. Improvements were seen across multiple domains and subdomains of the WFIRS-P. Functional improvements generally corresponded to improvements in symptoms. Children who were treatment responders were more likely to show significant functional improvements than non-responders.

Acknowledgments With great sadness, the authors wish to acknowledge the passing of Carla White, BSc, CStat, and recognize her contributions to this article. The authors acknowledge the assistance of Kavita Gajria and Jonathan Rubin, both formerly of Shire.

\section{Compliance with Ethical Standards}

Funding Clinical research and writing/editorial support was funded by the sponsor, Shire Development LLC. Under author direction, Wilson Joe, $\mathrm{PhD}$, of MedErgy, provided writing and editorial assistance for this publication. Shire provided funding to MedErgy for support in writing and editing this manuscript. Gina D'Angelo, PharmD, and Tom Babcock, DO, from Shire, also reviewed and edited the manuscript for scientific accuracy. Although the sponsor was involved in the design, collection, analysis, interpretation, and fact-checking of information, the content of this manuscript, the ultimate interpretation, and the decision to submit it for publication in CNS Drugs was made by the authors independently.

Conflicts of interest Mark A. Stein receives research support from Alcobra, Ironshore, Pfizer, and Shire, and is an advisor for Alcobra and Genco Life Sciences.

Vanja Sikirica, Brigitte Robertson, and Andrew Lyne are former employees of Shire, which funded this study, and owned stock/options in Shire at the time that the research was conducted.

Margaret D. Weiss has received research support from Purdue. She has served on speaker's bureaus for and received honoraria or consultant fees from Shire, Eli Lilly, Janssen, and Purdue.

Jeffrey H. Newcorn has received research support from Shire and Enzymotec. He has served as an advisor and/or consultant for Alcobra, BioBehavioral Diagnostics, Genco Sciences, Ironshore, Neos Therapeutics, Rhodes, Shire, Sunovion (DSMB), and the NFL.

Ethical Standards All human studies have been approved by the appropriate ethics committee and have therefore been performed in accordance with ethical standards laid down in the 1964 Declaration of Helsinki and its later amendments. All subjects gave their informed consent prior to their inclusion in the study.

Open Access This article is distributed under the terms of the Creative Commons Attribution-NonCommercial 4.0 International License (http://creativecommons.org/licenses/by-nc/4.0/), which permits any noncommercial use, distribution, and reproduction in any medium, provided you give appropriate credit to the original author(s) and the source, provide a link to the Creative Commons license, and indicate if changes were made.

\section{References}

1. Visser SN, Danielson ML, Bitsko RH, Holbrook JR, Kogan MD, Ghandour RM, et al. Trends in the parent-report of health care provider-diagnosed and medicated attention-deficit/hyperactivity disorder: United States, 2003-2011. J Am Acad Child Adoles Psychiatry. 2014;53(1):34-46.

2. American Academy of Pediatrics. Clinical practice guideline: diagnosis and evaluation of the child with attention-deficit/ hyperactivity disorder. American Academy of Pediatrics. Pediatrics. 2000;105(5):1158-70.

3. Stein MA, Szumowski E, Blondis TA, Roizen NJ. Adaptive skills dysfunction in ADD and ADHD children. J Child Psychol Psychiatry. 1995;36(4):663-70.

4. Parens E, Johnston J. Facts, values, and attention-deficit hyperactivity disorder (ADHD): an update on the controversies. Child Adolesc Psychiatry Ment Health. 2009;3(1):1.

5. Gathje RA, Lewandowski LJ, Gordon M. The role of impairment in the diagnosis of ADHD. J Atten Disord. 2008;11(5):529-37.

6. Gajria K, Livote E, Sikirica V, Reilly K, Erder MH. Validation of the Weiss Functional Impairment Rating Scale-Parent Report Form in attention deficit/hyperactivity disorder. Eur Neuropsychopharmacol. 2014;24(Suppl 2):S711.

7. Tarakçı̆̆ğlu MC, Cakin Memik N, Olgun NN, Aydemir O, Weiss MD. Turkish validity and reliability study of the Weiss Functional Impairment Rating Scale-Parent Report. Atten Defic Hyperact Disord. 2015;7(2):129-39.

8. Banaschewski T, Soutullo C, Lecendreux M, Johnson M, Zuddas A, Hodgkins P, et al. Health-related quality of life and functional outcomes from a randomized, controlled study of lisdexamfetamine dimesylate in children and adolescents with attention deficit hyperactivity disorder. CNS Drugs. 2013;27(10):829-40.

9. Fuentes J, Danckaerts M, Cardo E, Puvanendran K, Berquin P, De Bruyckere $\mathrm{K}$, et al. Long-term quality-of-life and functioning comparison of atomoxetine versus other standard treatment in pediatric attention-deficit/hyperactivity disorder. J Clin Psychopharmacol. 2013;33(6):766-74.

10. Baer S, Bogusz E, Green DA. Stuck on screens: patterns of computer and gaming station use in youth seen in a psychiatric clinic. J Can Acad Child Adolesc Psychiatry. 2011;20(2):86-94.

11. Hantson J, Wang PP, Grizenko-Vida M, Ter-Stepanian M, Harvey W, Joober R, et al. Effectiveness of a therapeutic summer camp for children with ADHD: Phase I Clinical Intervention Trial. J Atten Disord. 2012;16(7):610-7.

12. Stein MA, Waldman ID, Charney E, Aryal S, Sable C, Gruber R, et al. Dose effects and comparative effectiveness of extended release dexmethylphenidate and mixed amphetamine salts. J Child Adolesc Psychopharmacol. 2011;21(6):581-8.

13. Maziade M, Rouleau N, Lee B, Rogers A, Davis L, Dickson R. Atomoxetine and neuropsychological function in children with attention-deficit/hyperactivity disorder: results of a pilot study. J Child Adolesc Psychopharmacol. 2009;19(6):709-18.

14. Nagy P, Hage A, Coghill DR, Caballero B, Adeyi B, Anderson CS, et al. Functional outcomes from a head-to-head, randomized, double-blind trial of lisdexamfetamine dimesylate and atomoxetine in children and adolescents with attention-deficit/hyperactivity disorder and an inadequate response to methylphenidate. Eur Child Adolesc Psychiatry. 2015. [Epub ahead of print].

15. Biederman J, Melmed RD, Patel A, McBurnett K, Konow J, Lyne A, et al. A randomized, double-blind, placebo-controlled study of guanfacine extended release in children and adolescents with attention-deficit/hyperactivity disorder. Pediatrics. 2008;121(1): e73-84.

16. Sallee FR, McGough J, Wigal T, Donahue J, Lyne A, Biederman J. Guanfacine extended release in children and adolescents with attention-deficit/hyperactivity disorder: a placebo-controlled trial. J Am Acad Child Adolesc Psychiatry. 2009;48(2):155-65.

17. Wilens TE, Bukstein O, Brams M, Cutler AJ, Childress A, Rugino T, et al. A controlled trial of extended-release guanfacine and psychostimulants for attention-deficit/hyperactivity disorder. J Am Acad Child Adolesc Psychiatry. 2012;51(1):74-85.

18. Newcorn JH, Stein MA, Childress AC, Youcha S, White C, Enright $\mathrm{G}$, et al. Randomized, double-blind trial of guanfacine extended release in children with attention-deficit/hyperactivity 
disorder: morning or evening administration. J Am Acad Child Adolesc Psychiatry. 2013;52(9):921-30.

19. The Canadian Attention Deficit Hyperactivity Disorder Resource Alliance (CADDRA). Weiss Functional Impairment Rating Scale (WFIRS) instructions. http://www.caddra.ca/cms4/pdfs/caddra Guidelines2011WFIRSInstructions.pdf. Accessed June 252015.

20. The Canadian Attention Deficit Hyperactivity Disorder Resource Alliance (CADDRA). Weiss Functional Impairment Rating Scale-Parent Report (WFIRS-P). http://www.caddra.ca/cms4/ pdfs/caddraGuidelines2011WFIRS_P.pdf. Accessed June 25 2015.

21. Cohen J. A power primer. Psychol Bull. 1992;112(1):155-9.

22. Vidal R, Castells J, Richarte V, Palomar G, Garcia M, Nicolau R, et al. Group therapy for adolescents with attention-deficit/hyperactivity disorder: a randomized controlled trial. J Am Acad Child Adolesc Psychiatry. 2015;54(4):275-82.

23. Hervas A, Huss M, Johnson M, McNicholas F, van Stralen J, Sreckovic S, et al. Efficacy and safety of extended-release guanfacine hydrochloride in children and adolescents with attention-deficit/hyperactivity disorder: a randomized, controlled, phase III trial. Eur Neuropsychopharmacol. 2014;24(12): 1861-72.
24. Brod M, Pohlman B, Lasser R, Hodgkins P. Comparison of the burden of illness for adults with ADHD across seven countries: a qualitative study. Health Qual Life Outcomes. 2012;10:47.

25. Coghill D, Soutullo C, d'Aubuisson C, Preuss U, Lindback T, Silverberg M, et al. Impact of attention-deficit/hyperactivity disorder on the patient and family: results from a European survey. Child Adolesc Psychiatry Ment Health. 2008;2(1):31.

26. Holmberg K. Adolescent academic outcome of childhood attention-deficit/hyperactivity disorder-a population-based study. In: Norvilitis JM, editor. Contemporary Trends in ADHD Research. Rijeka: InTech; 2012.

27. Doshi JA, Hodgkins P, Kahle J, Sikirica V, Cangelosi MJ, Setyawan $\mathrm{J}$, et al. Economic impact of childhood and adult attention-deficit/hyperactivity disorder in the United States. J Am Acad Child Adolesc Psychiatry. 2012;51(10):990-1002.

28. Gjervan B, Torgersen T, Nordahl HM, Rasmussen K. Functional impairment and occupational outcome in adults with ADHD. J Atten Disord. 2012;16(7):544-52.

29. Dalsgaard S, Leckman JF, Mortensen PB, Nielsen HS, Simonsen $M$. Effect of drugs on the risk of injuries in children with attention deficit hyperactivity disorder: a prospective cohort study. Lancet Psychiatry. 2015;2(8):702-9. 